\title{
STRONGLY EXPOSED POINTS IN BOCHNER $L^{p}$-SPACES
}

\author{
PETER GREIM
}

\begin{abstract}
We give necessary and sufficient conditions for vector-valued $L^{p}$. functions to be strongly exposed in terms of their values $(1<p<\infty)$.
\end{abstract}

In this paper we give a characterization of the strongly exposed vector-valued $L^{p}$-functions in terms of their values $(1<p<\infty)$. In [4] J. A. Johnson has shown that, given a finite positive measure space $(\Omega, \Sigma, \mu)$, a Banach space $V$, an $x$ in $L^{p}(\mu, V)$ and a $g$ in $L^{q}\left(\mu, V^{\prime}\right)$ (where $1 / p+1 / q=1$ ), then $x$ is strongly exposed by $g$ if the scalar function $\|x(\cdot)\|$ is strongly exposed by $\|g(\cdot)\|$ and for almost all $t$ with $x(t) \neq 0$ the value $x(t)$ is strongly exposed by $g(t)$. He left the converse as an open question, but gave a kind of supplement in the case that $V$ has RNP. It is not too obvious that the converse should hold. Namely, a similar characterization of the extremal points is valid for separable $V$ (and Borel measures on Polish spaces [3]), but not in general [2].

We are going to show that the converse does hold for Radon measures $\mu$ on locally compact spaces, no matter what properties $V$ has (Theorem 2). If $V$ is separable we may even admit arbitrary positive measures $\mu$ (Theorem 1). In contrast to the extremal point situation the proof is rather simple.

In this manner we shall have a characterization of strong exposure as a relation between elements of $L^{p}(\mu, V)$ and $L^{q}\left(\mu, V^{\prime}\right)$; however, this is not yet a characterization of strongly exposed points. We shall give such a characterization under additional assumptions concerning $V$ (Theorems 3 and 4).

Recall that an element $x$ of a normed space $X$ is said to be strongly exposed by an element $\varphi$ of the dual $X^{\prime}$ if

(i) $\varphi x=\|\varphi\| \cdot\|x\| \neq 0$, and

(ii) each sequence $\left(x_{n}\right)$ in the ball with radius $\|x\|$, such that $\varphi x_{n}$ converges to $\varphi x$, converges to $x$ in norm.

For arbitrary functions $x: \Omega \rightarrow V$, and $g: \Omega \rightarrow V^{\prime}$ let us denote the functions $t \mapsto\|x(t)\|,\|g(t)\|$ and $g(t) x(t)$ by $|x|,|g|$ and $\langle x, g\rangle$, respectively. $\chi_{A}$ is the characteristic function of the subset $A$ of $\Omega$, and $\mathbf{v}$ is the constant function with value $v$. $B(v, \varepsilon)$ denotes the closed ball with center $v$ and radius $\varepsilon$.

Received by the editors May 5, 1982.

1980 Mathematics Subject Classification. Primary 46E40; Secondary 46B20.

Key words and phrases. Bochner $L^{p}$-space, Radon measure, separability, smoothness, strongly exposed point.

C1983 American Mathematical Society 0002-9939/82/0000-0834/\$01.75 
Before stating the theorems we make a few observations. Assume $g \in L^{q}\left(\mu, V^{\prime}\right)$ strongly exposes $x \in L^{p}(\mu, V)$. Then from

$$
\|g\| \cdot\|x\|=g x=\int\langle x, g\rangle d \mu \leqslant \int|g| \cdot|x| d \mu \leqslant\|g\| \cdot\|x\|,
$$

we deduce $\langle x, g\rangle=|g| \cdot|x|$ a.e. and, consequently, that $\chi_{A} g$ strongly exposes $\chi_{A} x$ for all measurable $A$ with $\chi_{A} x \neq 0$. Thus, in order to prove the theorems below, knowing that $x$ and $g$ vanish outside a suitable $\sigma$-finite set, we may assume without loss of generality that $\mu$ is finite. For the same reason we may assume that $|x|$ and $|g|$ are strictly positive. We may also assume w.l.o.g. that the scalars are real, since for complex scalars $\varphi$ strongly exposes $x$ if and only if the real part $\operatorname{Re} \circ \varphi$ of $\varphi$ strongly exposes $x$ in the underlying real space.

THEOREM 1. Let $(\Omega, \Sigma, \mu)$ be a positive measure space, $V$ a separable Banach space, $1<p<\infty, x \in L^{p}(\mu, V)$ and $g \in L^{q}\left(\mu, V^{\prime}\right)$. Then $g$ strongly exposes $x$ if and only if $|g|$ strongly exposes $|x|$ (i.e. $|g| /\|g\|=(|x| /\|x\|)^{p-1}$ ) and for almost all $t \in \Omega$, $g(t)$ strongly exposes $x(t)$ or $g(t)=0=x(t)$.

Proof. The "if" part is Theorem 1 in [4], where the finiteness of $\mu$ is an unnecessary restriction. Now let $g$ strongly expose $x$. From $\int|g| \cdot|x| d \mu=$ $\|g\| \cdot\|x\|$ and the uniform convexity of $L^{p}(\mu)$, it is clear that $|g|$ strongly exposes $|x|$. By the preceding remarks we can assume that $|x|$ and $|g|$ are strictly positive, $\mu$ is finite and the scalars are real. For $t \in \Omega$ and $n \in \mathbf{N}$ define the slices

$$
\begin{aligned}
& S(t, n):=\{v \in V|\|v\|<| x|(t), g(t) v>(1-1 / n) \cdot| g|(t) \cdot| x \mid(t)\}, \\
& d(t, n):=\min \{\varepsilon>0 \mid S(t, n) \subset B(x(t), \varepsilon)\},
\end{aligned}
$$

and

$$
e(t):=\inf \{d(t, n) \mid n \in \mathbf{N}\} .
$$

Observe that $g(t)$ strongly exposes $x(t)$ if and only if $e(t)=0$.

First we want to show that $e$ is a measurable function. By the definition of $e$ it is sufficient to show that the functions $d(\cdot, n)$ are measurable, i.e. the sets $\{t \mid d(t, n)$ $>\delta\}$ are measurable for all $\delta>0$. Fix such a $\delta$. For $v \in V$ define $A_{n, v}:=\{t \mid\|v\|$ $<|x|(t), g(t) v>(1-1 / n) \cdot|g|(t) \cdot|x|(t),\|x(t)-v\|>\delta\} . A_{n, v}$ is measurable since all the functions involved are measurable (w.l.o.g. $\mu$ is a complete measure). Now let $D$ be a countable dense subset of $V$. Observe that $d(t, n)>\delta$ if and only if $t \in A_{n, v}$ for a suitable $v \in D$. Thus we conclude that $\{t \mid d(t, n)>\delta\}$ is measurable as a countable union of measurable sets.

It remains to show that $\{t \mid e(t)>0\}$ has measure zero. Assume the contrary. Then there is a $\delta>0$ such that $A:=\{t \mid e(t)>\delta\}$ has positive measure. We want to construct a sequence $\left(y_{n}\right)$ in $L^{p}(\mu, V)$ such that $\left|y_{n}-x\right| \geqslant \delta$ on $A,\left|y_{n}\right| \leqslant|x|$ and $\left\langle y_{n}, g\right\rangle \geqslant(1-1 / n) \cdot|g| \cdot|x|$ a.e. Then $\left\|y_{n}\right\| \leqslant\|x\|,\left\|y_{n}-x\right\|^{p} \geqslant \mu(A) \cdot \delta^{p}$ and $g y_{n} \geqslant(1-1 / n) \cdot\|g\| \cdot\|x\|$, which means that $g$ does not strongly expose $x$, a contradiction. To this end let $n \in \mathbf{N}$ and define the sets $A_{n, v}$ as above. Then $A \subset\{t \mid d(t, n)>\delta\}=\cup_{v \in D} A_{n, v}$. Hence $A=\cup_{m=1}^{\infty} B_{m}$, where each $B_{m}$ is a measurable set contained in some $A_{n, v_{m}}, v_{m} \in D$. Obviously $y_{n}:=\chi_{\Omega \backslash A} x+\sum_{m=1}^{\infty} v_{m} \cdot \chi_{B_{m}}$ has the desired properties. 
THEOREM 2. Let $\mu$ be a Radon measure on a locally compact space $\Omega, V$ any Banach space, $1<p<\infty, x \in L^{p}(\mu, V)$ and $g \in L^{q}\left(\mu, V^{\prime}\right)$. Then $g$ strongly exposes $x$ if and only if $|g|$ strongly exposes $|x|$ and for almost all $t \in \Omega, g(t)$ strongly exposes $x(t)$ or $g(t)=0=x(t)$.

Proof. We proceed as in the proof of Theorem 1; we have to show that $\{t \mid e(t)>0\}$ is a null set. By Lusin's theorem the restrictions of $x$ and $g$ to suitable compact subsets, whose complements have arbitrarily small measures, are continuous. Thus we may assume w.l.o.g. that $x$ and $g$ are continuous on $\Omega$. Consequently the sets $A_{n, v}$ are open, and so is their union $\cup_{v \in V} A_{n, v}=\{t \mid d(t, n)>\delta\}$. This shows the measurability of $e$.

In order to verify that $\{t \mid e(t)>0\}$ is a null set, replace the set $A$ in the proof of Theorem 1 by a compact subset with positive measure $(A \subset\{t \mid e(t)>\delta\}, A$ compact, $\mu(A)>0)$ and proceed as above. Then by its compactness $A$ is contained in a finite union of sets $A_{n, v}$, and the functions $y_{n}$ defined analogously form the desired sequence.

Since the dual of $L^{p}(\mu, V)$ is $L^{q}\left(\mu, V^{\prime}\right)$ if $V^{\prime}$ has RNP, the following is an immediate corollary from the preceding theorems.

TheOREM 3. Let $V^{\prime}$ have RNP. Assume that $\mu$ is a Radon measure or $V$ is separable. Then for each strongly exposed $x \in L^{p}(\mu, V)$ almost all values $x(t)$ are strongly exposed or zero.

As mentioned before this is not yet a characterization of strongly exposed points in $L^{p}(\mu, V)$. Namely, given an $x \in L^{p}(\mu, V)$ such that almost all $x(t)$ are strongly exposed by some $g(t) \in V^{\prime}$, we do not know whether the $g(t)$ 's fit together in a measurable way. We do, however, if $V$ is smooth.

THEOREM 4. Let $V$ be smooth, $\mu$ arbitrary. Then each $x \in L^{p}(\mu, V)$ with $x(t)$ strongly exposed or zero a.e. is strongly exposed.

Proof. W.l.o.g. $\|x\|=1$. We may choose an $A \in \Sigma$ s.t. $\chi_{A} x=0$ and, for all $t \notin A, x(t)$ is strongly exposed by some norm 1 functional $g_{0}(t)$, and a sequence of simple functions $x_{n}$, vanishing on $A$ and taking only strongly exposed values outside $A$ (namely, certain $x(t)^{\prime}$ 's), such that $x_{n}(t) \underset{n}{\rightarrow} x(t)$ everywhere. Put $g_{0}(t):=0$ for $t \in A$. Since the support mapping $v \mapsto$ norm 1 functional supporting the unit ball in $v /\|v\|$ is norm- $\sigma\left(V^{\prime}, V\right)$-continuous on the unit sphere [1, p. 22], hence everywhere on $V \backslash\{0\}, g_{0}$ is the weak-* limit of a sequence of simple functions, hence weak-* measurable. From this it is easy to see that $\left\langle y, g_{0}\right\rangle$ is measurable for all $y \in L^{p}(\mu, V)$ and that $\varphi y:=\int\left\langle y,|x|^{p-1} g_{0}\right\rangle d \mu$ defines a linear functional on $L^{p}(\mu, V)$ with $\varphi x=1$ and $\|\varphi\|=1$. Although $g:=|x|^{p-1} g_{0}$ need not be Bochner measurable, $|g|$ is in $L^{q}(\mu)$ and the proof of [4, Theorem 1] shows that $\varphi$ strongly exposes $x$.

Added in proof. We can dispose of the RNP requirement in Theorem 3.

THEOREM 3'. Assume that $\mu$ is a Radon measure or $V$ is separable. Then for each strongly exposed $x \in L^{p}(\mu, V)$ almost all values $x(t)$ are strongly exposed or zero. 
This is a consequence of the facts that 1. any functional $\varphi$ on $L^{p}(\mu, V)$ may be regarded as a weak-* measurable function $g: \Omega \rightarrow V^{\prime}$ such that the upper integral $\int^{*}|g|^{q} d \mu$ equals $\|\varphi\|^{q}$ and $\varphi y=\int\langle y, g\rangle d \mu$ [5, p. 97], and 2. that Theorems 1 and 2 are valid also for these $g$ (where the measurability of $|g|$ is implicit in " $|g|$ strongly exposes $|x|$ ”). In order to verify the second fact recall that the proof of [4, Theorem 1] shows the sufficiency. If in the paragraph preceding Theorem 1 we replace $|g|$ by a measurable function $f \geqslant|g|$ such that $|g|^{q}$ and $f^{q}$ have the same (upper) integral, the arguments of this paragraph prove that $\langle x, g\rangle=|g| \cdot|x|=f$. $|x|$ a.e. and $f=0$ a.e. on $\{t|| x \mid(t)=0\}$. Consequently $|g|$ is measurable. But then (again assuming w.l.o.g. that $|x|$ and $|g|$ are strictly positive) the proofs of Theorems 1 and 2 work, since we only needed that the functions $\langle\mathbf{v}, g\rangle(v \in V)$ and $x$ and $|g| \cdot|x|$ are measurable.

REMARK. Although each strongly exposing $g$ has a measurable norm function $|g|$, it is not true that $g$ itself is measurable: as $L^{p}\left(\mu, l^{1}\right)$ has RNP, the strongly exposing functionals are dense in its dual which contains $L^{q}\left(\mu, l^{\infty}\right)$ as a proper closed subspace because $l^{\infty}$ lacks RNP ( $\mu$ not purely atomic).

\section{BIBLIOGRAPHY}

1. J. Diestel, Geometry of Banach spaces-Selected topics, Lecture Notes in Math., vol. 485, SpringerVerlag, Berlin, 1975.

2. P. Greim, An extremal vector-valued $L^{p}$-function taking no extremal vectors as values, Proc. Amer. Math. Soc. 84 (1982), 65-68.

3. J. A. Johnson, Extreme measurable selections, Proc. Amer. Math. Soc. 44 (1974), 107-112.

4. Strongly exposed points in $L^{p}(\mu, E)$, Rocky Mountain J. Math. 10 (1980), 517-519.

5. A. Ionescu-Tulcea and C. Ionescu-Tulcea, Topics in the theory of liftings, Springer-Verlag, Berlin, 1969.

Mathematisches Institut, Freie Universität, Arnimallee 2-6, D 1000 Berlin 33, Germany

Current address: Department of Mathematical Sciences, Memphis State University, Memphis, Tennessee 38152 